\title{
Younger age is associated with greater early neurocognitive decline postcardiopulmonary bypass
}

Kelsey Anderson, BA, ${ }^{\mathrm{a}}$ Olivia Ziegler, BA, ${ }^{\mathrm{a}}$ Guangbin Shi, MD, MSc, ${ }^{\mathrm{b}}$ Neel Sodha, MD, ${ }^{\mathrm{b}}$ Ian Ikeda, BS, ${ }^{\mathrm{a}}$ Jun Feng, MD, PhD, ${ }^{b}$ and Frank Sellke, $\mathrm{MD}^{\mathrm{b}}$

\section{ABSTRACT}

Objective: To examine the effect of aging on postoperative neurocognitive decline (NCD) in cardiac surgery patients.

Methods: Patients undergoing coronary artery bypass graft or open aortic valve replacement were administered the Repeatable Battery for the Assessment of Neuropsychological Status at preoperative, postoperative day (POD) 4, and 1 month. Blood samples were collected at preoperative, 6 hours postoperative, and POD 4. Plasma interleukin (IL)-6, tumor necrosis factor- $\alpha$, and C-reactive protein (CRP) levels were quantified. Quality of life was measured with the 12-Item Short Form Health Survey. Data were analyzed using paired ratio and unpaired $t$ tests with Welch's correction, and linear regression for cytokine levels.

Results: NCD occurred in 15 patients $(\mathrm{N}=33,45.5 \%)$. Dichotomized at age extremes ( $<60$ years; $\geq 75$ years), youngest patients had greater preoperative scores $(P=.02)$ with lower scores by POD $4(P=.03)$. There was no NCD in the oldest patients, and scores were not different between age groups on POD $4(P=.08)$. Regression at 1 month showed NCD scores again declined by age $(n=15)$, with younger scores returning toward baseline $(P=.008)$. Regression analyses showed decline by age at 6 hours postoperative and POD 4 in plasma CRP levels $(P=.05$ 6 hours, $P=.02$ POD 4). Dichotomizing IL- 6 levels by age ( $<70$ years, $\geq 70$ years) demonstrated that levels were greater in younger versus older patients at 6 hours postoperative $(P=.03)$, but not on POD 4 .

Conclusions: Younger patients tend to have better cognitive scores before surgery but scores at POD 4 are similar to those of older patients, with this trend disappearing at 1 month. IL- 6 and CRP upregulation is greater in younger patients, suggesting that a robust perioperative inflammatory response may be associated with reduction in neurocognitive function, and this may be greater in younger versus older patients. (JTCVS Open 2020;1:1-9)

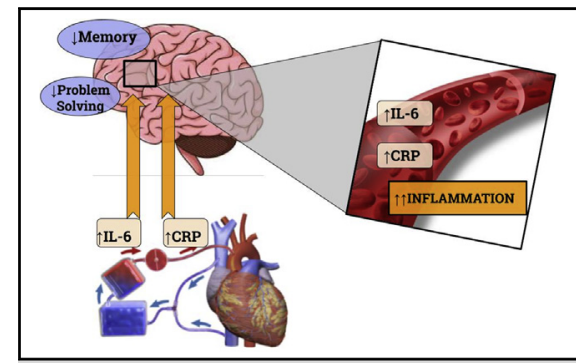

CPB upregulates IL- 6 and CRP in cerebral microvasculature, leading to cognitive decline.

\section{CENTRAL MESSAGE}

Younger age $(<60)$ is associated with greater perioperative NCD and inflammatory upregulation and poorer long-term QOL. This should be considered in the risks of cardiac surgery in younger patients.

\section{PERSPECTIVE}

Neurocognitive decline is a known risk of cardiac surgery, but its complex etiology has made it difficult to determine a definitive MOA or which populations are most at risk. Although younger patients may regain relatively greater cognition compared with older counterparts, potential for greater impact on QOL must be considered when assessing risks and timing of surgery.

See Commentary on page 10.
From the ${ }^{\mathrm{a}}$ Alpert Medical School of Brown University and ${ }^{\mathrm{b}}$ Division of Cardiothoracic Surgery, Cardiovascular Research Center, Rhode Island Hospital, Providence, RI.

This research project was mainly supported by R01-HL46716 to F.W.S., RO1HL128831 to F.W.S.; this work was supported in part by the National Institute of Health (NIH) 1R01HL127072, 1R01 HL136347, National Institute of General Medical Science (NIGMS) of the NIH (5P20-GM103652 [Pilot Project and CORE]), and AHA-Grant-in-Aid (\#15GRNT25710105) to J.F. and Brown University Medical Student Summer Research Award (K. A.).

Read at the 99th Annual Meeting of The American Association for Thoracic Surgery, Toronto, Ontario, Canada, May 4-7, 2019.
Received for publication Nov 6, 2019; revisions received Nov 6, 2019; accepted for publication Feb 3, 2020; available ahead of print April 9, 2020.

Address for reprints: Frank Sellke, MD, Division of Cardiothoracic Surgery, 2 Dudley St, MOC 360, Providence, RI 02905) (E-mail: fsellke@ lifespan.org). 2666-2736

Copyright $(C 2020$ by The Authors. Published by Elsevier Inc. on behalf of The American Association for Thoracic Surgery. This is an open access article under the CC BY-NC-ND license (http://creativecommons.org/licenses/by-nc-nd/4.0/). https://doi.org/10.1016/j.xjon.2020.02.001 


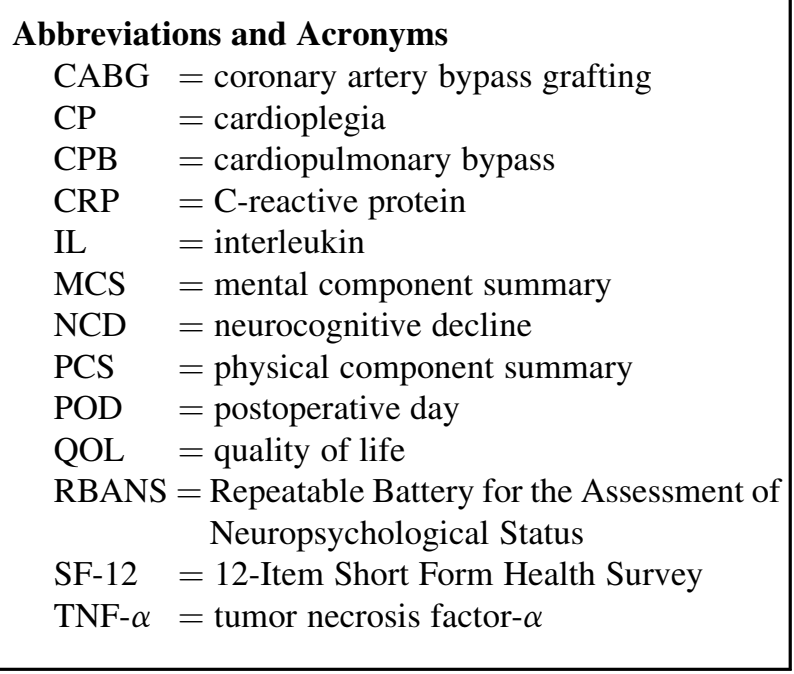

$\square$ Video clip is available online.

Cardiopulmonary bypass (CPB) allows patients to maintain blood flow and oxygenation during the cessation of cardiac activity. ${ }^{1}$ More than 350,000 people per year in the United States undergo $\mathrm{CPB}$ for coronary artery bypass grafting (CABG) procedures; however, $\mathrm{CPB}$ has been shown to be associated with neurocognitive decline (NCD) ${ }^{2,3}$ Studies report that $20 \%$ to $70 \%$ of patients who undergo CPB experience neurocognitive deficits, including delirium, cognitive decline, and neurobehavioral changes. ${ }^{4}$ Early cognitive function postoperatively has been shown to predict longterm functional outcomes. ${ }^{5}$ Therefore, early identification of impairment is essential to preventing further decline. Even with the advancement of surgical and anesthetic techniques, CPB outcomes have shown no significant improvement in the last decade.

Age is an established predictor of postoperative NCD, especially in patients older than 60 years. ${ }^{6}$ Specifically, age has been shown to be an independent predictor of decreased short-term memory function and cognitive impairment postoperatively. ${ }^{7}$ Furthermore, lower pre- and perioperative cognitive function has been associated with more rapid cognitive decline ${ }^{5}$ and poorer quality of life (QOL) postoperatively ${ }^{8}$; older patients are at increased risk of preoperative cognitive impairment and therefore greater postoperative risk. This effect is hypothesized to be mediated by neuroinflammatory reactions causing endothelial dysfunction ${ }^{6}$; however, no mechanism or specific inflammatory markers have been identified.

Surgery is a known cause of inflammatory upregulation contributing to possible NCD. In addition, studies have investigated the role of cardiac surgery with $\mathrm{CPB}$ in increasing systemic inflammation. ${ }^{9,10}$ We have previously demonstrated a correlation between inflammatory markers and NCD experienced post-CPB. Serum interleukin (IL)-8 levels were associated with a greater difference in neurocognitive function at postoperative day (POD) 4 compared with baseline. We use this preliminary to now expand our study to additional cytokines to further evaluate the inflammatory response. Because CPB causes increased inflammatory upregulation, we believe its use may exacerbate the extent of postoperative NCD. The aim of this study is to assess the effect of age on the expression of these inflammatory markers and short-term cognitive decline post-CPB.

\section{METHODS \\ Patient Enrollment}

This study was approved by the Lifespan IRB (date and number of approval: original approval date: March 10, 2010; newest approval date April 11, 2019. Internal reference number: 004410) and consent was obtained. Patients older than 18 years scheduled to receive CABG, aortic valve replacement, mitral valve replacement, or a combination of these procedures at Rhode Island Hospital from July 2016 to September 2018 were considered for enrollment in our single-institution, prospective cohort study. All procedures used CPB. Preoperative risk was calculated using the Society of Thoracic Surgeons score. Patients were excluded if they had hepatic disease (abnormal liver function tests, cirrhosis), stroke within 1 year, severe neurologic deficits (subjective, or diagnosis of dementia), chronic renal failure $(\mathrm{Cr}>2.0)$, high-grade carotid stenosis $(>70 \%)$, heavily calcified aorta (determined by available imaging, chart review), or severely impaired vision/blindness. Patients undergoing aortic arch or root procedures or receiving combined carotid/CABG procedures were not included. All patients were native English speakers.

\section{Surgical Technique}

Patients underwent induction of anesthesia and invasive monitoring. A midline sternotomy or right anterior mini-thoracotomy (1 patient) was performed per standard technique. Operations were performed by 1 of 4 experienced cardiac surgeons. All patients underwent systemic heparinization (activated clotting time $>400$ seconds). The CPB circuit consisted of an Affinity (Medtronic, Minneapolis, Minn) integrated hollow fiber oxygenator/ cardiotomy reservoir with trillium coating (Medtronic), and an arterial 38$\mathrm{mg}$ filter with trillium coating. The cardioplegia perfusion system (Medtronic Myotherm 4:1 system) with trillium coating was used. A hypothermic blood-based cardioplegia (CP) solution $\left(8^{\circ} \mathrm{C}, 4: 1\right.$ mixture of oxygenated blood and hyperkalemic crystalloid solution; CAPS, Lanham, $\mathrm{Md})$ was used. An initial 650 to $1000 \mathrm{~mL}$ of hyperkalemic $\left(\mathrm{K}^{+} 25 \mathrm{mmol} / \mathrm{L}\right)$ $\mathrm{CP}$ solution was delivered antegrade into the aortic root, followed by 200 to $500 \mathrm{~mL}$ of $\mathrm{CP}$ solution antegrade or retrograde $\left(\mathrm{K}^{+} 8 \mathrm{mmol} / \mathrm{L}\right)$ every 15 to 20 minutes until the crossclamp was removed. After recovery of cardiac function, patients were ventilated and separated from CPB and managed in the usual manner.

\section{Neurocognitive Assessment}

Patients were administered a neurocognitive battery consisting of Repeatable Battery of Assessment of Neurological Status (RBANS), Beck's Depression Inventory-II, Cognitive Difficulties Scale, and the 12Item Short Form Health Survey (SF-12). The battery was administered twice: (1) before surgery during the outpatient presurgical appointment or inpatient preoperative admission period, and (2) POD 4 during inpatient admission. POD 4 time point was chosen based on literature ${ }^{10}$ and the 
ability to re-evaluate patients while still admitted and accessible. All patients were treated by the same team of caregivers and given comparable narcotic pain regimens to control for the differential effects of sleep deprivation and narcotics on NCD. In addition, patients were subjectively evaluated for level coherence and were excluded from analysis if deemed unfit to reliably test.

The RBANS measures 5 neurocognitive domains: immediate memory, delayed memory, attention, language, and visuospatial skills. The battery consists of a standard series of activities. RBANS provides 2 different versions (A and B) of their forms to prevent confounding by long-term recall. All batteries were administered several weeks apart, with form A first and form B second. All assessments were scored by the study team member who administered the assessment. Total scaled score was based on a normal distribution (score range: $40-160,50$ th percentile $=100$ ) that considered age, sex, level of education, and form version administered. An individual subscore for each of the 5 testing domains was also calculated. As per previously defined standards, ${ }^{11}$ a decrease in score by 8 points between testing periods was considered significant (confidence interval $=90 \%$ ) NCD.

Phone interviews were performed March to August of 2019 using the SF-12 survey to assess postoperative QOL. It measures physical (physical component summary [PCS]) and mental (mental component summary [MCS]) health as composite scores that can be compared with agestratified population means. A score lower than the population mean by 6.97 (PCS) or 6.24 (MCS) is considered significant (95\% confidence interval). All interviews were conducted $>9$ months postoperatively; therefore, all patients are expected to have fully recovered from surgery at time of interview.

\section{Blood Collection and Analysis}

Peripheral intravenous blood collection was performed at 3 time points: (1) preoperatively immediately before surgery, (2) 6 hours postsurgery, and (3) POD 4 at the same time as neurocognitive assessment. Approximately $10 \mathrm{cc}$ of blood was collected each time. Blood was collected in standard

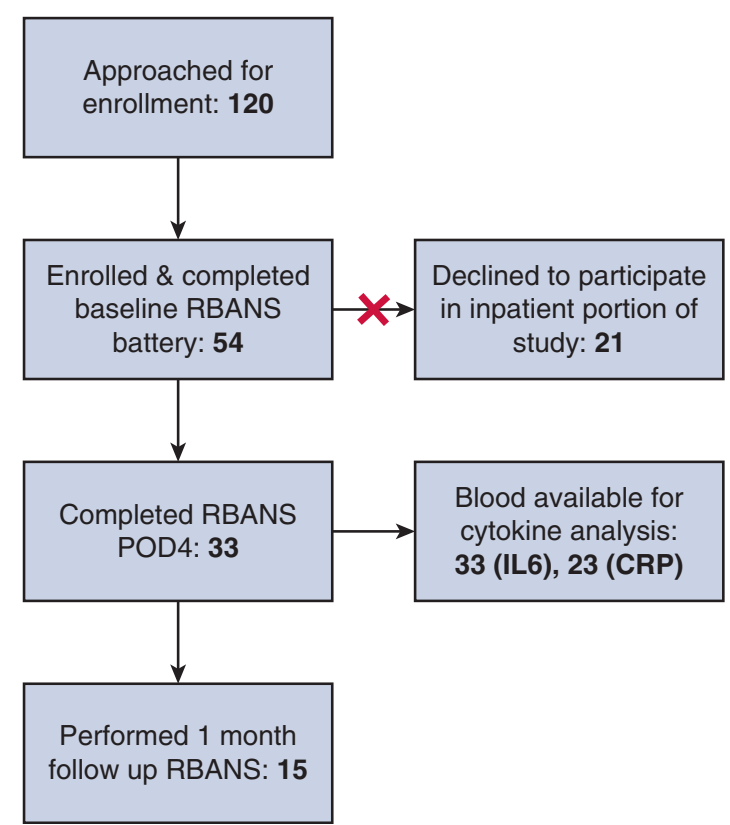

FIGURE 1. Patient flow chart demonstrating patient enrollment (54), dropout (21), and inclusion in neurocognitive (33) and inflammatory marker analysis (23). RBANS, Repeatable Battery for the Assessment of Neuropsychological Status; $P O D$, postoperative day; $I L$, interleukin; $C R P$, C-reactive protein. ethylenediaminetetraacetic acid and PAXgene tubes and stored on ice for immediate transport (approximately 30 minutes). Whole blood samples were centrifuged at $4^{\circ} \mathrm{C}$ at $2000 \mathrm{rpm}$ for 20 minutes. Supernatant plasma was distributed to $1-\mathrm{mL}$ aliquots and stored at $-80^{\circ} \mathrm{C}$.

Plasma IL-6, tumor necrosis factor- $\alpha$ (TNF- $\alpha$ ), and C-reactive protein (CRP) levels were quantified using human high-sensitivity in vitro enzyme-linked immunosorbent assay kit (Abcam, Cambridge, United Kingdom) per the manufacturer's instructions. These cytokines were selected because of their potency and acute presentation during inflammation. We have examined these cytokines in the past and have detected a correlation with $\mathrm{NCD}{ }^{10}$

\section{Statistical Analysis}

Total RBANS scaled scores were compared at age extremes (youngest: $<60$ years, $\mathrm{N}=6$; oldest: $\mathrm{N}>75$ years, $\mathrm{N}=5$ ). All other neurocognitive data were analyzed dichotomized by age $<70$ years, $\mathrm{N}=17$ versus age $\geq 70$ years, $\mathrm{N}=16$ ) RBANS scores and cytokine data dichotomized by age group were compared using paired and unpaired ratio $t$ tests with Welch's correction. Cytokine levels $(\mathrm{N}=23)$ were analyzed by foldchange from baseline and 6 hours. Linear regression was used to analyze graded trends by age, NCD, and cytokine levels. $P<.05$ was considered statistically significant.

\section{RESULTS}

Fifty-four patients were enrolled in the study (consented and completed baseline NC testing), and $33(61.1 \%)$ were included in final analysis at POD 4 (Figure 1). Twentyone patients withdrew their participation from the study after initial enrollment and did not complete all components of the study. Table 1 includes patient demographic characteristics. The majority of the study sample was male $(72.4 \%)$, white $(94.0 \%)$, and had less than a college degree $(60.1 \%)$. The mean age was 68.1 years $( \pm 8.6)$.

Table 2 demonstrates clinical characteristics and operative variables of our study sample dichotomized by age $<70$ years and age $\geq 70$ years. Patient groups differed only by Society of Thoracic Surgeons score.

\section{Neurocognitive Decline}

Significant postoperative NCD occurred in 15 patients $(45.5 \%)$, with $7(46.7 \%)$ in the older group and 8 $(53.3 \%)$ the younger group suffering decline. When dichotomized at age extremes (youngest: $<60$ years, $\mathrm{N}=6$; oldest: $>75$ years, $\mathrm{N}=5$ ), the youngest patients had significantly greater baseline total neurocognitive scores before surgery $(100.8,14.6)$ compared with the oldest patients $(81.0,10.4)(P=.02)$. Youngest patients experienced a significant decrease in total scores between baseline and POD $4(P=.04)$. Surprisingly, there was no significant decrease in total scores between baseline and POD 4 for oldest patients $(P=.09)$, and scores were not significantly different between the 2 age groups at POD $4(P=.08)$ (Figure 2). When stratifying by the 5 RBANS subscore domains, there were no significant differences by age ( $<70$ years vs $\geq 70$ years) in any individual domain. Linear regression analyses showed significantly greater scores in younger patients at preoperatively $(P=.008)$, but no 
TABLE 1. Patient demographic characteristics

\begin{tabular}{lc}
\hline Demographics $(\mathbf{N}=\mathbf{3 3})$ & $\mathbf{n}(\%)$ \\
\hline Sex $($ male $)$ & $24(72.7)$ \\
Age, y, mean (SD) & $68.1( \pm 8.6)$ \\
Race & \\
White & $31(94.0)$ \\
African American & $1(3.0)$ \\
Native American & $1(3.0)$ \\
\hline
\end{tabular}

$S D$, Standard deviation.

significant trend by age at postoperative $(P=.26)$ (Figure 3). Only 2 patients in our sample received isolated aortic valve replacement; excluding these patients from analysis does not significantly affect our overall results.

Data for 15 patients in our sample were available at 30 days postoperative. Linear regression of this subset analyzing age versus neurocognitive score showed that scores significantly declined with age $(P=.008)$ at this more distant time point.

\section{Depression, Cognition, and QOL}

Beck's Depression Inventory showed mean preoperative scores of 6.88 in the young age group and 7.0 in the older age group. At POD 4, scores were 8.86 in the young age group and 8.09 in the older age group. There were no significant differences in score by age or time point.

The Cognitive Difficulties Scale showed mean preoperative scores of 32.88 in the young age group and 37.53 in the older age group. At POD 4, scores were 33.93 in the young age group and 25.57 in the older age group. There were no significant differences in score by age or time point.
A total of 17 patients were available by phone to complete the SF-12 QOL survey. All interviews were performed with the primary patient except for one, which was completed by the patient's daughter. Of the 15 surveyed, $6(35.3 \%)$ were age $<70$ years and 11 $(64.7 \%)$ were $\geq 70$ years old. PCS and MCS scores were calculated for each patient. Among young patients, $2(40 \%)$ had PCS scores significantly lower than the age average, and none had significantly lower MCS scores. Among older patients, $3(33.3 \%)$ demonstrated PCS scores significantly lower than average, and $2(20.0 \%)$ demonstrated significantly lower MCS scores. There was no significant difference in lower PCS scores between age groups $(P=.18)$. Subjective trends showed that although not all reached the designated threshold below the mean, a greater proportion of younger patients $(\mathrm{N}=4,66.7 \%)$ had PCS scores lower than the age mean compared to older patients $(\mathrm{N}=6,54.5 \%)$.

\section{Blood Inflammatory Markers}

Inflammatory markers IL-6, CRP, and TNF- $\alpha$ were measured in 23 patients. Compared with baseline preoperative levels, IL-6 and CRP levels were significantly greater at 6 hours postoperative and POD $4(P<.01)$. IL- 6 levels were greatest at 6 hours postoperative and significantly greater than levels at POD $4(P<.01)$.

Analyzed by age, regression analysis showed that change in plasma CRP levels declined significantly by age at both 6 hours $(P=.05)$ and POD $4(P=.02)$, with greatest change occurring in the youngest patients (Figure 4 ). There was no linear trend on regression analysis of IL- 6 and TNF- $\alpha$ levels

TABLE 2. Clinical variables by age

\begin{tabular}{|c|c|c|c|}
\hline Characteristic & Age $\geq 70$ y $(n=16)$ & Age $<70$ y $(n=17)$ & $P$ value \\
\hline Sex, male, $\mathrm{n}(\%)$ & $11(68.8 \%)$ & $13(76.5 \%)$ & .62 \\
\hline Age, y, mean (SD) & $74.3( \pm 4.8)$ & $62.1( \pm 7.0)$ & $<.01 *$ \\
\hline Diabetes, n (\%) & $9(56.3 \%)$ & $9(52.9 \%)$ & .85 \\
\hline Preoperative A1C, mean (SD) & $6.4( \pm 0.9)$ & $6.8( \pm 2.2)$ & .23 \\
\hline STS score, mean (SD) & $4.6( \pm 4.4)$ & $1.3( \pm 1.0)$ & $<.01 *$ \\
\hline Time on $\mathrm{CPB}$, min, mean (SD) & $107.8( \pm 73.3)$ & $81.3( \pm 84.9)$ & .10 \\
\hline ICU LOS, d, mean (SD) & $4.7( \pm 4.9)$ & $4.1( \pm 3.4)$ & .34 \\
\hline Hypertension, n (\%) & $16(100.0 \%)$ & $16(94.1 \%)$ & .90 \\
\hline Hypercholesterolemia, n (\%) & $16(100.0 \%)$ & $17(100.0 \%)$ & 1.0 \\
\hline Hemoglobin, g/dL, mean (SD) & $13.4( \pm 2.2)$ & $14.2( \pm 1.7)$ & .13 \\
\hline Hematocrit, \%, mean (SD) & $40.3 \pm .5)$ & $42.2( \pm 4.4)$ & .17 \\
\hline Procedure, $\%$ CABG, mean (SD) & $14(87.5 \%)$ & $15(88.2 \%)$ & .95 \\
\hline CVA & $0(0.0 \%)$ & $0(0.0 \%)$ & 1.0 \\
\hline Mortality & $0(0.0 \%)$ & $0(0.0 \%)$ & 1.0 \\
\hline
\end{tabular}

$S D$, Standard deviation; $S T S$, Society of Thoracic Surgeons; $C P B$, cardiopulmonary bypass; $I C U$, intensive care unit; $L O S$, length of stay; $C A B G$, coronary artery bypass grafting; CVA, cerebrovascular accident. *Statistical significance in this study defined as $P<.05$. 
Total RBANS Score at Baseline and POD4, by Age

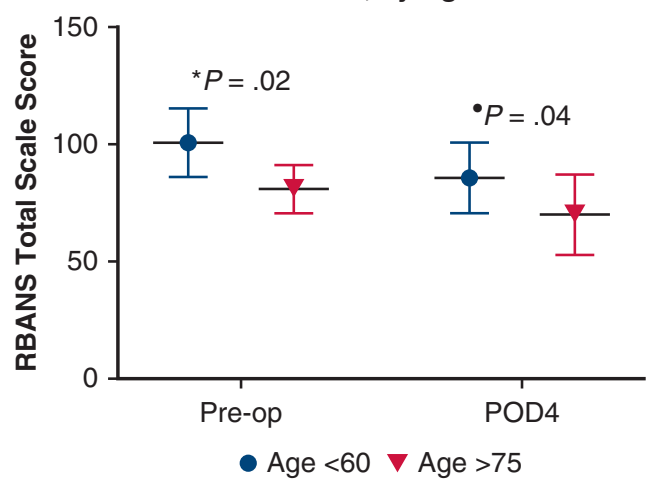

FIGURE 2. $t$-test analysis showing that younger patients have greater neurocognitive scores at baseline $(P=.02)$, with significantly lower scores at POD $4(P=.04)$; however, at POD 4 their scores are the same as older patients. Older patients did not have significantly decreased neurocognitive scores at POD 4. Age is dichotomized as $<60$ and $>75$ in this diagram to show trend without clustering along the median age. RBANS, Repeatable Battery for the Assessment of Neuropsychological Status; POD, postoperative day.

by age. Dichotomizing IL- 6 levels by age group $(<70$ years, $\geq 70$ years) and analysis by comparison of means demonstrated that IL-6 levels were significantly greater in younger patients at 6 hours postoperatively $(P=.03)$ compared with older patients, but this trend disappeared by POD 4 . There were no significant changes in TNF- $\alpha$ levels by age or time point. Analysis of inflammatory changes at 6 hours by presence versus absence of NCD at POD 4 did not show a significant difference in levels between groups (IL-6, $P=.15$; CRP, $P=.38$ ).

Linear regression analyzing 6-hour IL-6 and CRP level versus NCD at POD 4 showed no significant trend in graded response ( $P=.75, .15$, respectively).

\section{DISCUSSION}

In this study, we found that younger age is associated with both greater NCD and a greater peri- and postoperative inflammatory response. These results support our hypothesis that upregulation of the inflammatory response during this period is related to immediate postoperative neurocognitive function. This is summarized in our Figure 5. This perioperative period is crucial, as perioperative cognitive decline has been shown to predict greater long-term cognitive decline and less recovery of function compared with patients who do not experience significant $\mathrm{NCD}^{5,11,12}$ However, although a correlation between some inflammatory markers and NCD was found, a causal relationship is not proven (Video 1).

Interestingly, the results of our study diverge from literature claiming that older age yields worse cognitive outcomes postcardiac surgery. ${ }^{11-13}$ In our study, younger patients tended to have better cognitive scores before surgery, but their scores at POD 4 were the same as older patients. This may be explained by the fact that in the immediate postoperative period, cognitive function declines in most patients. Then, as the literature suggests, younger patients experience greater cognitive improvement in the long term versus older patients. In addition, our "younger" patients, defined by the general functional and health status of our patient population, fell within the classification of "elderly" in some studies, explaining why our effect may not agree with some other literature. ${ }^{14,15}$ Our 1-month analyses also confirm literature stating that $\mathrm{NC}$ function tends to return in younger patients with time. Finally, there is even evidence of this cognitive delay postoperatively in populations as young as pediatric patients, indicating that this phenomenon is not limited to older patients, no matter how they are defined. ${ }^{16}$

Ultimately, it is important to note that this initial perioperative cognitive decline does occur in younger patients, and its presence may affect future ability to return to
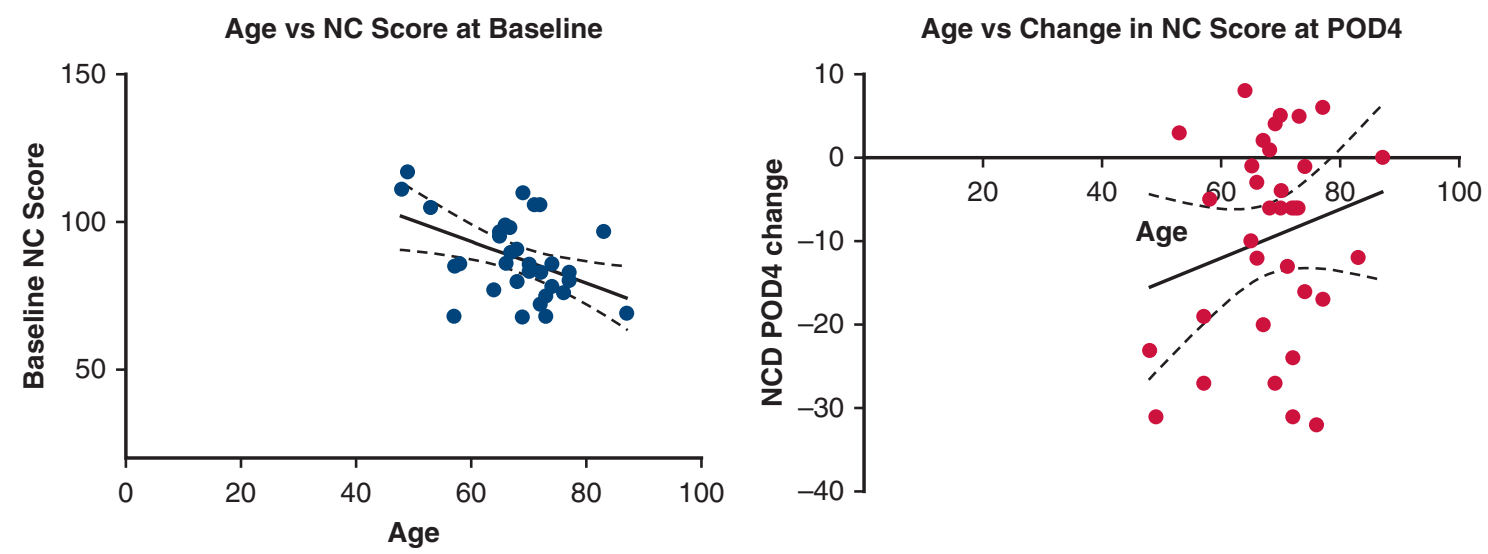

FIGURE 3. Linear regression analysis (includes all age data points) demonstrating that at baseline preoperative testing, NC score declines with age $(P=.008)$. At POD 4 , this trend disappears $(P=.26)$. NC, Neurocognitive; $P O D$, postoperative day. 


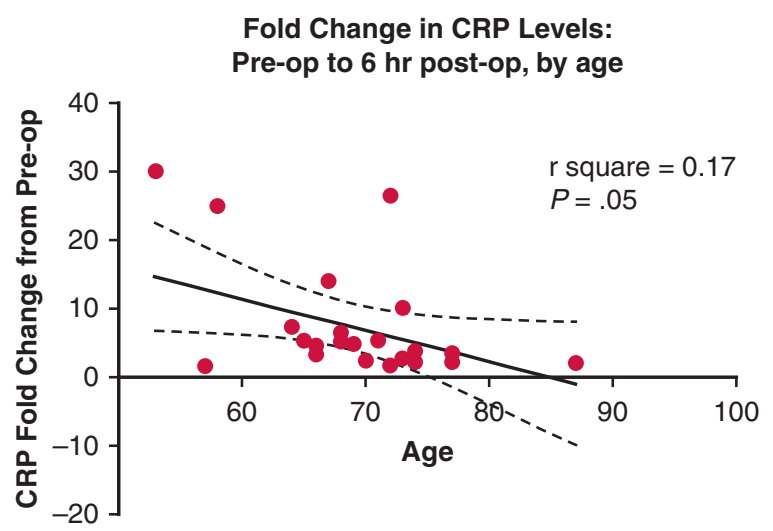

FIGURE 4. Linear regression of CRP levels at 6 hours postoperatively by age shows a significant downward trend with age, indicating that older patients experience a smaller degree of CRP upregulation in the perioperative period. ( $\mathrm{N}=23, P=.05)$. CRP, C-reactive protein.

baseline cognitive function in the long term. Therefore, although younger patients may have relatively better outcomes than elderly patients, it is important to consider that this perioperative NCD may significantly affect future QOL, as discussed to follow.

We consider that the RBANS battery is not sensitive enough to measure differential changes in neurocognitive function in the perioperative period; however, if this were true, we would expect mean scores at POD 4 in both groups to fall closer to the RBANS's minimum value of 40 . These data may instead be explained by the fact that younger patients, starting at greater baseline scores, have a higher potential drop in score postsurgery than older patients.

Recently, the Partner-3 trial demonstrated greater cerebrovascular accident risk with surgical aortic valve replacement versus transcatheter aortic valve replacement cases, raising the question if patients in our study are also at embolic risk and which risk factors are modifiable. Although we do not investigate this in our current study, we acknowledge that this is a likely component of the complex mechanism causing postoperative NCD. One study showed that optimizing mental health and instrumental activities of daily living function preoperatively was the only potentially "modifiable" risk factors associated with improved recovery of cognitive function. ${ }^{7}$

\section{Cytokine Analysis}

As expected, an upregulation in the inflammatory response was seen among all patients postoperatively. Generally, IL-6 levels rose robustly and acutely by 6 hours and declined toward baseline by POD 4. CRP levels continued to rise through POD 4 for all patients, maintaining the inflammatory response through our second neurocognitive testing point. Linear regression analysis did not find a significant trend in cytokine levels by NCD; however, this does not negate the presence of any effect, rather only a graded relationship Instead of a graded phenomenon, it is possible that NCD occurs once cytokines reach a certain

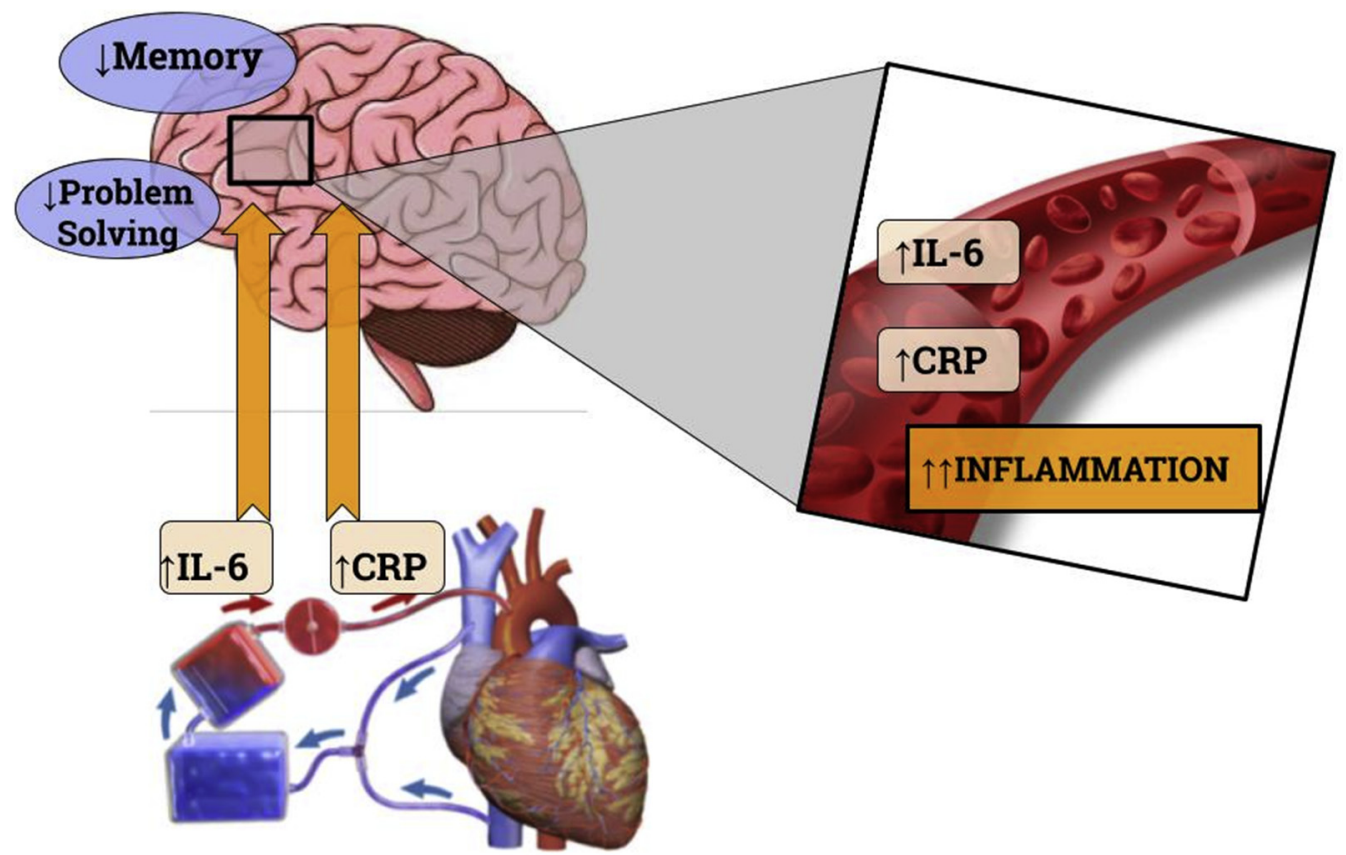

FIGURE 5. Our proposed mechanism that cardiopulmonary bypass exacerbates the increase in IL- 6 and CRP levels caused by surgery, leading to inflammatory changes in the cerebral microvasculature resulting in decreased cognitive function postsurgery. This mechanism is not proposed in isolation but rather as a component of the complex postoperative neurocognitive decline mechanism. $I L$, interleukin; $C R P$, C-reactive protein. 


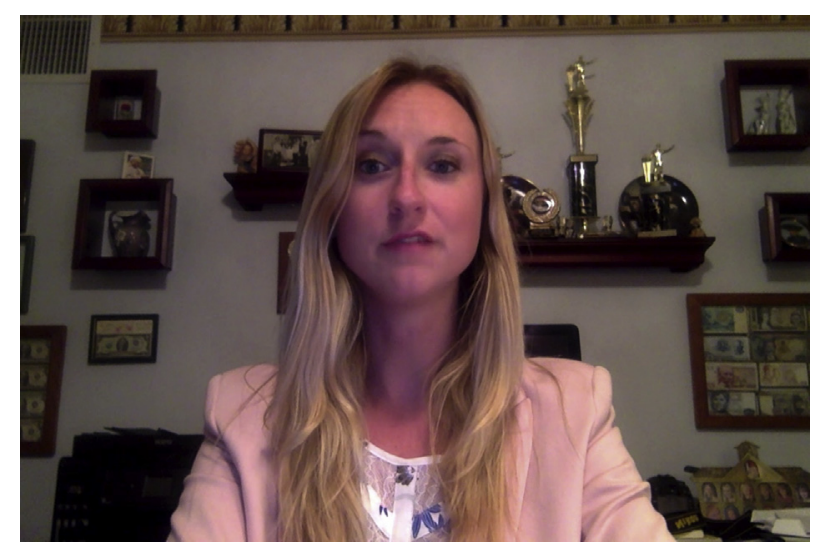

VIDEO 1. First author Kelsey Anderson describes the hypothesized mechanism, results, and implications of the study conclusions on younger patients considering elective surgical intervention using cardiopulmonary bypass. Video available at: https://www.jtcvs.org/article/S2666-2736(20) 30003-6/fulltext.

threshold. Of note, the trillium coated perfusion system used has been shown to mildly in increase inflammatory upregulation postoperatively, but this should not significantly alter our intergroup results, as it was used with all patients. ${ }^{14}$

Overall, there was a more robust peri- and postoperative inflammatory response seen in younger patients. This is consistent with the fact that while age is associated with a low-grade inflammatory state,, , 13,14 upregulation of the inflammatory response and microvascular blood flow tends to decline with age. ${ }^{15-19}$ In addition, studies have shown that when comparing postoperative elderly patients who developed NCD versus those who did not, there was no difference in preoperative inflammatory markers between groups. ${ }^{18,20}$ Evaluated with our current study, this may mean that although elderly patients do experience NCD, systemic inflammation may be a more direct mechanism in younger versus older patients. However, although a correlation of expression of inflammatory cytokines was found with neurocognitive decline in this study and other studies, a causal relationship was not established.

\section{Postsurgical QOL}

Depression is a significant confounder of cognitive function, especially among elderly patients. ${ }^{19}$ In both age groups and time points, mean scores were between 0 and 13, which indicates minimal depression level. In addition, degree of depression did not differ significantly by age, indicating that depression levels did not significantly influence the results of our study.

The SF-12 assesses general self-reported health, not changes specific to cardiac surgery. Therefore, these results must be interpreted understanding that patients vary in the degree of complexity and chronicity of their comorbid conditions, and that it would be impossible to quantify how much cardiac surgery directly affected QOL. However, a subjectively greater degree of lower physical health was present in younger patients. Younger patients would be expected to be less chronically and complexly ill. This is an important finding, indicating that while literature may indicate that overall cognitive status of younger patients may improve move than in older patients, they still experience lower QOL as a long-term postsurgical outcome. In addition, because younger patients are expected to have fewer comorbid conditions, the effect of cardiac surgery on overall QOL becomes proportionally greater than in older patients.

Together, these data argue that younger patients do experience neurocognitive decline postoperatively, and, importantly, experience a greater decline from baseline than older patients. Our data suggest that this may be linked to their more robust perioperative inflammatory response. Early cognitive dysfunction has been linked to worse QOL. ${ }^{21}$ This may be significant when discussing risks of elective cardiac surgery to this younger patient population who generally experience greater baseline cognitive function and therefore potentially greater QOL.

\section{Limitations}

Our study is limited by a small sample size predominantly composed of a white, male population. This small sample size, and the fact that this is a single-center study, limits generalizations that can be drawn by our significant results. We are reassured that the effect we see is not primarily due to sampling error, given that there were significant differences seen between groups preoperatively and both groups experienced subjective (although not both statistically significant) decline.

Patients included in the study may be healthier than the general population undergoing open cardiac surgery, as those included met additional exclusion criteria to participate in the study; however, these patients are of particular interest to this research, as they may suffer greater decline in QOL specifically due to surgical complications compared with complex patients who are already managing complications from comorbid conditions.

\section{Future Directions}

Continuing analysis at later time points in the postoperative period (eg, 4 weeks, 6 months) is important for evaluating trends in neurocognitive function, potential recovery, or lack thereof beyond the initial perioperative period. These additional data would provide insight into the effects of the degree of inflammatory response on long-term cognitive function and its subsequent clinical significance. In addition, since embolic phenomenon are a potentially important component in the pathology of $\mathrm{NCD}$; we plan to pursue imagining studies to evaluate the effects of this process. 


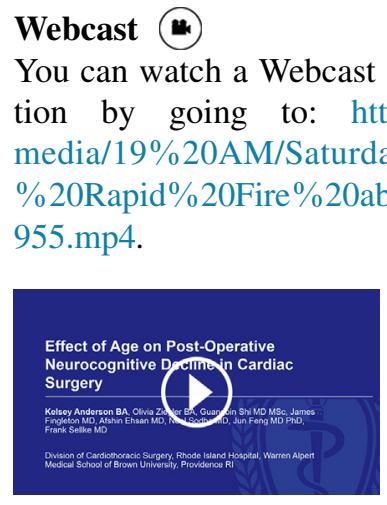

\section{Conflict of Interest Statement}

The authors reported no conflicts of interest.

The Journal policy requires editors and reviewers to disclose conflicts of interest and to decline handling or reviewing manuscripts for which they may have a conflict of interest. The editors and reviewers of this article have no conflicts of interest.

We thank all the nurses, physician assistants, and perfusionists working in cardiac surgery at Rhode Island Hospital for collecting the blood samples and recording patient characteristics. We also thank the nurses and physician assistants at Division of Cardiac Surgery of Rhode Island Hospital for collecting patient consent forms.

\section{References}

1. Passaroni AC, de Morales Silva MA, Yoshida WB. Cardiopulmonary bypass: development of John Gibbon's heart-lung machine. Braz J Cardiovasc Surg. 2015;30:235-45.

2. Bakaeen FG, Chu D, Kelly RF, Holman WL, Jessen ME, Ward HB. Perioperative outcomes after on- and off-pump coronary artery bypass grafting. Tex Heart Inst J. 2014;41:144-51.

3. Ahonen J, Salmenperä M. Brain injury after adult cardiac surgery. Acta Anaesthesiol Scand. 2004;48:4-19.

4. Stroobant N, Van Nooten G, Van Belleghem Y, Vingerhoets G. The effect of CABG on neurocognitive functioning. Acta Cardiol. 2010;65:557-64.

5. Inouye SK, Marcantonio ER, Kosar CM, Tommet D, Schmitt EM, Travison TG, et al. The short-term and long-term relationship between delirium and cognitive trajectory in older surgical patients. Alzheimers Dement. 2016;12:766-75.

6. Riedel B, Browne K, Silbert B. Cerebral protection: inflammation, endothelial dysfunction, and postoperative cognitive dysfunction. Curr Opin Anaesthesiol. 2014;27:89-97.

7. Newman MF, Croughwell ND, Blumenthal JA, Lowry E, White WD, Spillane W, et al. Predictors of cognitive decline after cardiac operation. Ann Thorac Surg. 1995;59:1326-30.

8. Newman MF, Grocott HP, Mathew JP, White WD, Landolfo K, Reves JG, et al. Report of the substudy assessing the impact of neurocognitive function on quality of life 5 years after cardiac surgery. Stroke. 2001;32:2874-81.

9. Sodha NR, Clements RT, Sellke FW. Vascular changes after cardiac surgery: role of NOS, COX, kinases, and growth factors. Front Biosci (Landmark Ed). 2009; 14:689-98.

10. Ramlawi B, Rudolph JL, Mieno S, Khabbaz K, Sodha NR, Boodhwani M, et al. Serologic markers of brain injury and cognitive function after cardiopulmonary bypass. Ann Surg. 2006;244:593-601.

11. Wilk CM, Gold JM, Bartko JJ, Dickerson F, Fenton WS, Knable M, et al. Testretest stability of the repeatable battery for the assessment of neuropsychological status in schizophrenia. Am J Psychiatry. 2002;159:838-44.
12. Xie G, Zhang W, Chang Y, Chu Q. Relationship between perioperative inflammatory response and postoperative cognitive dysfunction in the elderly. Med Hypotheses. 2009;73:402-3.

13. Moller J, Cluitmans P, Rasmussen LS, Houx P, Rasmussen H, Canet J, et al. Long-term postoperative cognitive dysfunction in the elderly: ISPOCD1 study. Lancet. 1998;351:857-61.

14. Nemeth E, Vig K, Racz K, Koritsanszky KB, Ronkay KI, Hamvas FP, et al. Influence of the postoperative inflammatory response on cognitive decline in elderly patients undergoing on-pump cardiac surgery: a controlled, prospective observational study. BMC Anesthesiol. 2017;17:113.

15. Załęska-Kocięcka M, Skrobisz A, Woźniak S, Greszata L, Dąbrowski M, Grabowski M, et al. Patterns of changes in functional and neurocognitive status in elderly patients after transcatheter vs. surgical aortic valve replacements. Minerva Anestesiol. 2018;84:328-36.

16. Gunn JK, Beca J, Hunt RW, Goldsworthy M, Brizard CP, Finucane K, et al. Perioperative risk factors for impaired neurodevelopment after cardiac surgery in early infancy. Arch Dis Child. 2016;101:1010-6.

17. Hoel TN, Videm V, Baksaas ST, Mollnes TE, Brosstad F, Svennevig JL. Comparison of a Duraflo II-coated cardiopulmonary bypass circuit and a trillium-coated oxygenator during open-heart surgery. Perfusion. 2004;19:177-84.

18. Vasto S, Candore G, Balistreri CR, Caruso M, Colonna-Romano G, Grimaldi MP, et al. Inflammatory networks in ageing, age-related diseases and longevity. Mech Ageing Dev. 2007;128:83-91.

19. Ashcroft GS, Horan MA, Ferguson MW. Aging alters the inflammatory and endothelial cell adhesion molecule profiles during human cutaneous wound healing. Lab Invest. 1998;78:47-58.

20. Lemstra AW, Kalisvaart KJ, Vreeswijk R, van Gool WA, Eikelenboom P. Preoperative inflammatory markers and the risk of postoperative delirium in elderly patients. Int J Geriatr Psychiatry. 2008;23:943-8.

21. Phillips-Bute B, Mathew JP, Blumenthal JA, Grocott HP, Laskowitz DT, Jones RH, et al. Association of neurocognitive function and quality of life 1 year after coronary artery bypass graft (CABG) surgery. Psychosom Med. 2006;68:369-75.

Key Words: neurocognitive decline, cardiothoracic surgery, age, inflammation, CPB

\section{Discussion}

Dr Rakesh C. Arora (Winnipeg, Manitoba, Canada). Thank you for very interesting paper. Questions, please.

Dr Kathleen C. Clement (Baltimore, $M d$ ). There's a great talk and seeing that you're a medical student, congratulations. My name is Kathleen Clem and I work at Johns Hopkins. So, when you analyze your data, it looks like you just analyzed greater than 70 versus less than 70. Did you ever look at like a sensitivity analysis to look at specific age groups or use a continuous variable to see what were the predictive factors for that outcome you're looking at?

Kelsey Anderson (Providence, RI). Thank you. That's a great question. Right now, the end of the study is limited by a fairly small size. It's about an end of 33 , which because of the nature of the study and how many follow-up time points we have, it unfortunately limits us from doing a more specific age-based analysis by those discrete time points and outcomes.

However, the goal of the study is to expand that number and that for the specific neurocognitive scores. We did look at age extremes to get a little bit more away from that 
the crowding around at age 70 that could, you know, "Merc up" the results. So we looked at patients younger than 65 and older than I believe 75 so we had a little bit more of a diversification in those patients.

Dr Carlos A. Mestres (Zurich, Switzerland). So, do you think then because for the younger patients that isn't happening? Regulation of cytokines. Do you think that if in the future we should start talking about cytokines depletion through something like hemo-absorption, which is just coming up, for instance, in acute inflammation like an endocarditis or even an acute aortic dissection, you think is something that we need to look into for the specific dental patients?

Anderson. I think for the younger patients, especially because they are presenting presumably with fewer comorbidities that doing what we can to reduce this inflammation in this time point and especially because of potentially increases quality of life more specifically would be important.

Dr Arora. Okay Thank you very much. 